\title{
Viromes of one year old infants reveal the impact of birth mode on microbiome diversity
}

\author{
Angela McCann ${ }^{1,2}$ ， Feargal J Ryan ${ }^{1,2}$ ， Stephen R Stockdale ${ }^{1,2,3}$, Marion Dalmasso ${ }^{1}$, Tony Blake ${ }^{1,2}$, C.

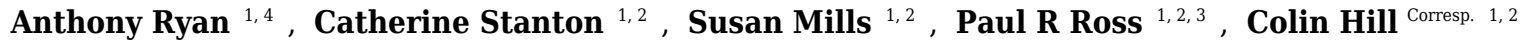 \\ ${ }^{1}$ APC Microbiome Institute, Cork, Cork, Ireland \\ 2 School of Microbiology, University College Cork, Cork, Cork, Ireland \\ 3 Teagasc Food Research Centre, Fermoy, Cork, Ireland \\ 4 Department of Neonatology, Cork University Maternity Hospital, Cork, Cork, Ireland \\ Corresponding Author: Colin Hill \\ Email address: c.hill@ucc.ie
}

Establishing a diverse gut microbiota after birth is being increasingly recognised as important for preventing illnesses later in life. It is well established that bacterial diversity rapidly increases post-partum; however, few studies have examined the infant gut virome/phageome during this developmental period. We performed a metagenomic analysis of 20 infant faecal viromes at 1 year of age to determine whether spontaneous vaginal delivery (SVD) or caesarean section (CS) influenced viral composition. We find that birth mode results in distinctly different viral communities, with SVD infants having greater viral and bacteriophage diversity. We demonstrate that CrAssphage is acquired early in life, both in this cohort and two others, although no difference in birth mode is detected. A previous study has shown that bacterial OTU's (operational taxonomic units) identified in the same infants could not discriminate between birth mode at 12 months of age. Therefore, our results indicate that vertical transmission of viral communities from mother to child may play a role in shaping the early life microbiome, and that birth mode should be considered when studying the early life gut virome. 
1 Viromes of one year old infants reveal the impact of birth mode on microbiome diversity

2 Angela McCann*,1,3, Feargal J. Ryan*,1,3, Stephen R. Stockdale*,1,2,3, Marion Dalmasso ${ }^{1,3, \dagger}$, Tony

3 Blake $^{1,3}$, C. Anthony Ryan ${ }^{1,4}$, Catherine Stanton ${ }^{1,3}$, Susan Mills ${ }^{1,3}$, Paul R. Ross ${ }^{1,2,3}$, Colin Hill ${ }^{1,3, *}$

$4 \quad{ }^{1}$ APC Microbiome Institute, University College Cork, Co. Cork, Ireland

$5 \quad{ }^{2}$ Teagasc Food Research Centre, Moorepark, Fermoy, Co. Cork, Ireland

$6 \quad{ }^{3}$ School of Microbiology, University College Cork, Cork, Ireland

$7 \quad{ }^{4}$ Department of Neonatology, Cork University Maternity Hospital, Co. Cork, Ireland

$8 \uparrow$ Present address: Normandie Univ, UNICAEN, ABTE, 14000 Caen, France

$9 \quad *$ These authors contributed equally to this work.

10 Corresponding author: Colin Hill

11 c.hill@ucc.ie 
19

20

21

22

\section{Abstract}

Establishing a diverse gut microbiota after birth is being increasingly recognised as important for preventing illnesses later in life. It is well established that bacterial diversity rapidly increases post-partum; however, few studies have examined the infant gut virome/phageome during this developmental period. We performed a deep-sequencing metagenomic analysis of 20 infant faecal viromes at 1 year of age to determine whether spontaneous vaginal delivery (SVD) or caesarean section (CS) influenced viral composition. We find that birth mode results in distinctly different viral communities, with SVD infants having greater viral and bacteriophage diversity. We demonstrate that CrAssphage is acquired early in life, both in this cohort and two previously published studies of the infant virome, although no difference in birth mode is detected. A previous study has shown that bacterial OTU's (operational taxonomic units) identified in the same infants could not discriminate between birth mode at 12 months of age. In conclusion, our results indicate that vertical transmission of viral communities from mother to child may play a role in shaping the early life microbiome, and that birth mode should be considered when studying the early life gut virome.

\section{Introduction}

The human gut microbiota is a diverse community densely populated with bacteria, archaea, protists, fungi, and viruses. Studies focused on gut bacteria suggest that healthy individuals are characterised by high species diversity (Heiman \& Greenway, 2016), with compositional alterations and decreased diversity linked to conditions such as obesity, diabetes and inflammatory bowel disease (IBD) (Imhann et al 2016, Karlsson et al 2013, Ley et al 2005). 
41 Gut microbiota colonization in infants is a critical process, characterised by initial low bacterial

42 diversity which increases with time such that by 1 year of age the microbiota converges towards

43 that of an adult and fully resembles an adult microbiota by 2-5 years of age (Rodriguez et al 2015).

44 Several factors have been shown to influence an infant's microbiota, from birth mode to antibiotic usage, diet, geographical location, lifestyle and age (Milani et al 2017, Rodriguez et al 2015). Indeed, Hill et al. confirmed that delivery mode and gestational age significantly influence bacterial composition in the infant gut during the first 24 weeks of life (Hill et al 2017).

The gut virome is an area of growing interest with relation to the microbiota (Breitbart et al 2003, Minot et al 2013) and gut virome alterations have been recorded between healthy and diseased states; for instance, an increase in the taxonomic richness of Caudovirales has been associated with IBD (Norman et al 2015). However, there is a significant knowledge gap about healthy human viral populations, with large portions of the sequence data from human virome metagenomic studies representing uncharacterised viruses either not present in current databases or described using in silico methods without host information or taxonomic assignment (Krishnamurthy and Wang 2017). Reyes et al. (2010) demonstrated intrapersonal virome variation between adult twins over a one year period was low, while interpersonal variation was high (Reyes 57 et al 2010). Manrique et al (2016) demonstrated the presence of a healthy human phageome, which is a collection a bacteriophage which are present in a large portion of healthy individuals and hypothesized that this community plays a key role in the structure of the human microbiome and by extension human health. Research on the gut virome in infancy and early life ( $0-3$ years) thus

61 far has exclusively focused on longitudinal studies in twin pairs (Lim et al 2015, Reyes et al 2015) or has been based on a single infant (Breitbart et al. 2008). Reyes et al. (2015) identified different

63 viral assembly stages of the gut microbiota in 20 healthy infant twin pairs (0-3 years) and revealed 
64 that this program of assembly is impaired in twins discordant for severe acute malnutrition (Reyes

65 et al 2015). Lim et al. (2015) conducted a longitudinal study of the virome and bacterial

66 microbiome in 4 twin pairs, from birth to 2 years, and revealed that the expansion of the bacterial

67 microbiome with age was accompanied by a contraction and shift in the bacteriophage composition

68 (Lim et al 2015). The work conducted by Lim et al. was unsuited to examining the impact of birth

69 mode as only a single twin pair in that study was born by standard vaginal delivery. Reyes et al

70 did not report on the birth mode of the infants in their study; however, as many of the twin pairs

71 in that study were discordant for forms of malnutrition and received a dietary intervention as a

72 result, measuring the impact of birth mode in those infants would be complicated by confounding

73 factors. Thus, to date, no investigation has had a study design allowing for direct investigation of

74 the impact of birth mode on diversity and composition of the virome in early life but birth mode

75 has been proposed as a putative modulator of microbiome diversity (Milani et al 2017). Therefore,

76 we performed a deep-sequencing metagenomic examination of faecal DNA viromes of 20 infants

77 at 1 year of age and investigated whether spontaneous vaginal delivery (SVD) or caesarean section

78 (CS) influenced gut virome composition and diversity.

Materials \& Methods

\section{Selection of faecal samples}

82 Faecal samples for all infants were collected as part of the INFANTMET (Hill et al 2017) study.

83 Infant guardians were approached for written consent between February 2012 and May 2014 in

84 Cork University Maternity Hospital, with ethical approval provided by the Cork University

85 Hospital Research Ethics Committee. Ethical approval reference: ECM (w) 07/02/2012. In order 
86 to control for potential variants, faecal samples were randomly chosen from those available that

87 best met the following criteria: (1) an equal number of Spontaneous Vaginal Delivered (SVD) and

88 emergency Caesarean-Section (CS) infants, (2) gestational-term matched infants, (3) age matched infants, and (4) a balanced number of breastfed versus bottle-fed infants from SVD and CS available samples. In addition, technical criteria included the availability of $>1 \mathrm{~g}$ of starting faecal material. As a result, 20 infant faecal samples were selected; 10 were from SVD infants and 10 were from CS infants. Of the 10 infant faecal samples per cohort, 7 and 8 of the CS and SVD delivered infants, respectively, were breastfed. For details related to samples chosen in this study, see Supplementary Table 1.

\section{Preparation of faecal viral suspensions}

Viruses were separated from faecal solids using the following method. Faeces $(0.5 \mathrm{~g})$ was suspended in $10 \mathrm{ml}$ of SM buffer (50 mM Tris-HCl; $100 \mathrm{mM} \mathrm{NaCl} ; 8.5 \mathrm{mM} \mathrm{MgSO}$; pH 7.5). Samples were homogenised by vortexing for $5 \mathrm{~min}$, before centrifuging twice at 4,075 $\mathrm{x}$ for 10 min at $4{ }^{\circ} \mathrm{C}$ in a swing-bucket centrifuge to remove large particulates and bacterial cells. Faecal viral suspensions were filtered twice through a $0.45 \mu \mathrm{m}$ pore diameter filter and processed immediately for DNA extraction.

\section{Extraction of viral DNA}

Preparation of viral suspensions and DNA extractions were optimised for the small faecal samples collected from infant adsorbent nappies. To viral suspensions, $\mathrm{NaCl}$ (final conc. $0.5 \mathrm{M}$ ) and $10 \%$ (w/v) polyethylene glycol (PEG; average molecular weight 8000) were dissolved before samples were chilled on ice for $3 \mathrm{hrs}$. Viruses were then precipitated from solution in a $4{ }^{\circ} \mathrm{C}$ pre-chilled centrifuge at $4,075 \times \mathrm{g}$ in a swing-bucket centrifuge for $20 \mathrm{~min}$. The viral-PEG pellet was 
108 suspended in $400 \mu \mathrm{L}$ of SM buffer, viruses were then separated from the PEG by treating the 109 samples with an equal volume of chloroform, vortexing for $30 \mathrm{sec}$, and centrifuging at 2,500 x g. 110 Clarified viral preparations were treated with $20 \mathrm{U}$ of DNase I and $10 \mathrm{U}$ of RNase I (final 111 concentrations; Ambion) for $1 \mathrm{hr}$ at $37^{\circ} \mathrm{C}$, after the addition of $40 \mu \mathrm{L}$ of $10 \mathrm{x}$ Nuclease Buffer $(50$ $112 \mathrm{mM} \mathrm{CaCl} 2 ; 10 \mathrm{mM} \mathrm{MgCl} 2$ ). Nucleases were inactivated at $70{ }^{\circ} \mathrm{C}$ for $10 \mathrm{~min}$ before samples were 113 treated with $20 \mu \mathrm{L}$ of $10 \%$ SDS and $2 \mu \mathrm{L}$ of freshly prepared $20 \mathrm{mg} / \mathrm{ml}$ Proteinase K for $20 \mathrm{~min}$ 114 at $56^{\circ} \mathrm{C}$. Remaining intact viruses were lysed by the addition of $100 \mu \mathrm{L}$ of Phage Lysis Buffer (4.5 $115 \mathrm{M}$ guanidine thiocyanate; $45 \mathrm{mM}$ sodium citrate; $250 \mathrm{mM}$ sodium lauroyl sarcosinate; $562.5 \mathrm{mM}$ $116 \beta$-mercaptoethanol; $\mathrm{pH} 7.0$ ) with incubation at $65^{\circ} \mathrm{C}$ for $10 \mathrm{~min}$. Viral DNA was purified by two 117 treatments with an equal volume of phenol:chloroform:isoamyl alcohol $(25: 24: 1)$ and passing the 118 resulting purified DNA through a QIAGEN Blood and Tissue Purification Kit and eluting samples 119 in $50 \mu \mathrm{L}$ of TE Buffer.

\section{Viral DNA amplification, library preparation and sequencing}

Infant faecal viral DNA concentrations were equalised before amplification for sequencing using an Illustra GenomiPhi V2 kit (GE Healthcare). Amplifications of purified viral DNA were performed in triplicate on all samples as described by the manufacturer. Subsequently, an equal volume of each amplification and an equal volume of the original viral DNA purification were pooled together for paired-end Nextera XT library preparation (Illumina) as described by the manufacturer. Metagenomic sequencing of stool filtrates was performed using the Illumina MiSeq

127 (Illumina Inc. U.S.A) by generating 300 bp paired-end read libraries following the manufacturer's instructions. 
130 Voided infant faeces were suspended in 1:20 (w/v) SM buffer, centrifuged twice at 4,075 x g for

$13110 \mathrm{~min}$ at $4{ }^{\circ} \mathrm{C}$, before filtering twice through $0.45 \mu \mathrm{m}$ pore diameter filters. A $200 \mu \mathrm{L}$ aliquot of

132 the faecal viral-enriched suspension was lysed using a QIAGEN Blood and Tissue Purification Kit

133 following the manufacturer's recommendations with elution in $50 \mu \mathrm{L}$ of TE Buffer. The

134 concentration of double stranded viral DNA was calculated using a Qubit 3.0 Flurometer (Life

135 Technologies) using a Qubit dsDNA High Sensitivity Assay Kit (Thermo Fisher). Subsequently, 136 all dsDNA concentrations were normalised to $0.05 \mathrm{ng} / \mu 1$. The choice of primers and conditions 137 for detecting pan-human associated TTV by qPCR were as described by Ssemadaali et al. (2016) 138 , using SensiFAST SYBR No-ROX mastermix and a LightCycler 480 thermocycler. A two-fold 139 serial dilution of the purified TTV PCR product was also included in the qPCR for standard curve 140 analysis.

\section{Analysis of virome sequencing data}

\section{Metagenomic analysis}

144 The quality of the raw reads was visualized with FastQC v0.11.3. Nextera adapters were removed with Cutadapt v1.9.1 (Martin 2011) followed by read trimming and filtering with Trimmomatic v0.36 (Bolger et al 2014) to ensure a minimum length of 60 , maximum length of 150 , and a sliding

147 window that cuts a read once the average quality in a window size of 4 falls below a Phred score of 30. Levels of Bacterial contamination were estimated by classifying reads with SortMeRNA v2.0 (Kopylova et al 2012) against the SILVA database and by aligning reads against the cpn60db (Hill et al 2004) with bowtie2 in end-to-end alignment mode (Langmead and Salzberg 2012).

151 Reads were then assembled with the metaSPAdes assembler (Nurk et al 2017). 
152 Virome sequence reads were classified into known viral orders and families using the Kaiju

153 metagenomic classifier (Menzel et al 2016) and the NCBI non-redundant protein database (NCBI

154 Resource Coordinators, 2018). The number of Torque Teno virus (TTV) homologues was counted

155 by predicting genes from all contigs with Prodigal (Hyatt et al 2010), and then by BLASTp search 156 against ORF1 from known TTV genomes (Hsiao et al 2016). Prototypical crAssphage was 157 downloaded from GenBank using accession number NC_024711.1 (Dutilh et al 2014). Assembled 158 contigs, in this study and from the Reyes et al. dataset, with similarity to crAssphage were detected 159 by BLAST homology (Reyes et al., 2015). Complete, or near complete, crAss-like genomes (96160 $99 \mathrm{~kb}$ ) were compared using the 'Pyani' program (https://github.com/widdowquinn/pyani), implementing the ANIm method with a 500bp window size. The pyani percentage identity 162 comparison calculations were exported to $\mathrm{R}$ and graphed using the gplot 'heatmap.2' package. 163 GenBank files of the 6 crAss-like phages were generated and used to visualise whole genome 164 comparisons by EasyFig v2.2.2 (Sullivan et al 2011), using a minimum Blast length of 50bp and 165 identity of 30bp.

\section{Statistical analyses}

167 16S OTU tables from the INFANTMET (Hill et al 2017) cohorts were obtained and used in this study to examine the connection between the virome and the bacteriome. In order to account for partially assembled viruses, abundances were correlated and those with a Spearman correlation of greater than 0.9 were grouped into a single feature. All statistical analyses were performed in $\mathrm{R}$ v3.3.0 (Team 2000). Alpha diversity metrics including Chao1 richness and Shannon index were computed with PhyloSeq v1.16.2 (McMurdie and Holmes 2013) and plotted with ggplot2 v2.2.1

173 (Wickham 2016). Between-group differences in alpha diversity were tested with a Mann-Whitney 174 test (also known as a two sample Wilcoxon test). Unweighted Bray-Curtis distance was used as 
175 input for a Principle Coordinates Analysis (PCoA) as performed by the pcoa function in the ape

176 package v4.1. Adonis tests were performed using the vegan package v2.4.3 (Oksanen et al 2007)

177 in to test community level differences. Differential abundance analyses for both virome and 16S

178 rRNA datasets was carried out with DESeq2 (Love et al 2014) based upon the previous reporting

179 that it has increased sensitivity on datasets with less than 20 samples per group (Weiss et al 2017).

\section{Results \& Discussion}

697,558 following strict quality control, making this the deepest sequenced infant virome dataset to date (Lim et al 2015, Reyes et al 2015). Paired end sequence reads were classified against the nr database from NCBI using Kaiju (Menzel et al 2016) which translates reads into 6 possible read frames for classification based on amino acid homology (Fig 1a). As with previous published viral taxonomic group (Norman et al 2015, Reyes et al 2015), with a mean $46.59 \%$ unclassified reads per sample in this cohort (Fig 1a). For viruses which were classifiable, it was only possible to do so at higher taxonomic ranks. The most abundant viruses detected were Caudovirales, Microviridae and Anelloviridae (Fig 1a), in agreement with previously published published findings (Lim et al 2015). The number of sequence reads classifiable as Anelloviridae, a family of single stranded DNA vertebrate viruses, showed a large difference between birth modes (Fig 1a, Wilcox test, $\mathrm{p}=0.02$ ). The Anelloviridae and specifically their type species, Torque Teno Virus

195 (TTV), have been characterised by a very high prevalence in humans worldwide, although their 196 host interaction remains poorly understood (Spandole et al 2015). Previous research of the infant 
197 virome to date have described the Anelloviridae as important but variable members of the gut

198 virome in the first years of life. Lim et al reported Anelloviridae peak in abundance between 6 and

19912 months of age and that infants harbour multiple Anelloviridae species (Lim et al 2015). Reyes

200 et al reported that the abundance of the Anelloviridae decreases after 15 months of age and that

201 members of this family were able to discriminate twin pairs discordant for malnutrition (Reyes et

202 al 2015). Further investigation of Anelloviridae in this cohort found that the richness of TTV was

203 significantly increased in infants delivered by vaginal birth (Fig 1b), but not by breastfeeding status

204 (Fig S1). Similar observations of vertical transmission of TTV have previously been reported,

205 although it has been unclear whether this transmission occurs in the birth canal or in the post-

206 partum period through mother-infant contact such as breast feeding (Tyschik et al 2017).

207 Transmission of TTV to infants could occur at any point during their development through

208 environmental exposure, contact with other infants or parental contact. Given the ubiquity of

209 Anelloviridae throughout humans (Spandole et al 2015) it seems likely that transmission can

210 happen through multiple routes and forms of contact but based on the difference observed here it

211 would suggest that vertical transmission is one such route. As multiple displacement amplification

212 is known to distort the abundance of ssDNA viruses such as TTV (Roux et al 2016), we sought to

213 verify these results using quantitative PCR on the unamplified DNA from the infant faecal

214 samples. However, due to limited sample material this was possible for only $50 \%$ of the samples

215 (Table S2). The abundance of detected TTV DNA was found to be significantly higher in the SVD

216 cohort over the CS group (Wilcox test, $\mathrm{p}=0.048$ ) with no difference detected by breastfeeding

217 status (Wilcox test, $\mathrm{p}=0.49$ ).

218

CrAssphage is a highly abundant constituent of the human gut microbiome (Dutilh et al

219 2015) and has previously been suggested as not present in the early life microbiome (Lim et al 
220 2015). However, we recovered several complete crAssphage genomes from infants, both in this

221 cohort and from virome assemblies examining the gut virome of Malawian infants (Fig 1c,

222 Supplementary figure 3) (Reyes et al. 2015). These crAssphage genomes showed high levels of

223 nucleotide homology and syntenty to the prototypical crAssphage genome as originally described

224 by Dutilh et al with average nucleotide identity between all six crass genomes here between $95 \%$

225 and 97\% (Fig 1c, Fig S2). The impact of this highly abundant and prevalent bacteriophage on the

226 stability of the gut microbiota is thus far unknown, but crAssphage was recently described as just

227 one member of a previously unknown but expansive bacteriophage family (Yutin et al 2017).

228 CrAssphage is thought to predate on bacteria within the phylum Bacteroidetes (Dutilh et al 2014,

229 Yutin et al 2017), which is a constituent of the infant microbiome from as early as one week after

230 birth (Hill et al 2017).

In order to assess the full diversity of the DNA virome, further analysis was based on the abundance of contigs assembled with MetaSPAdes. Estimates of 16S rRNA and $60 \mathrm{kDa}$ chaperone protein (cpn60), two commonly used bacterial phylogenetic markers, and comparison to shotgun metagenomic samples from the Human Microbiome Project indicated that all samples contained low levels of bacterial contamination (Fig S3 and Table S3). However in order to avoid any bacterial sequence being considered for analysis only contigs which passed the VirSorter virome “decontamination" mode (Roux et al 2015), contained genes that corresponded to at least one

238 known Prokaryotic Virus Orthologous Groups (pVOGs) (Grazziotin et al 2016) or showed nucleotide homology to a known virus in the nt database (Coordinators 2016) were used for further analysis. This resulted in a total of 2028 assembled contigs (Table S4) being taken forward for analysis, out of a possible 5629 , which recruited a median of $64.075 \%$ of reads per sample. There

242 was no difference detected in the percentage of reads recruited between birth mode groups (Wilcox 
243 test, $p=0.57)$. Close to half of these assemblies $(925,45.1 \%)$ bore no homology at any length to

244 anything in the nt database with an E-value cutoff of 1e-5, highlighting the lack of viral

245 representation in current databases (Krishnamurthy and Wang 2017). The largest assembled

246 sequence included in the analysis a $146 \mathrm{~kb}$ which had a best hit of 126 bases at 88.1 percent identity

247 to a region of Lachnoclostridium sp. YL32 annotated as a transfer RNA. Of the 2028 included

248 contigs only 36 were detected as circular by VirSorter (Table S4). Alpha and beta diversity

249 analyses identified significant differences between infant viromes by birth mode (Figure 2a,c, Fig

250 S4a). Differences in bacterial diversity at 1 year of age was not observed with the 16S rRNA

251 sequencing data (Figure 2b,d, Fig S4b). The lack of taxonomic resolution with the 16S rRNA gene

252 possibly masks diversity differences at the species, or strain level which may only be observable

253 through shotgun metagenomic sequencing (Yarza et al 2014).

254 No single virus, or viral taxon, was identified as being universally absent in CS and 255 universally present in SVD. However, DESeq2 did identify 32 contigs differentially abundant by 256 birth mode, including TTV and several contigs bearing high levels of nucleotide homology to 257 Bifidobacteria temperate phages including those from Bifidobacterium longum subsp. infantis and 258 subsp. longum (Table S5) being increased in infants born by SVD. This may be reflective of 259 differential colonisation of Bifidobacterium by birth mode, an observation which is supported by 260 16S rRNA sequence based studies (Hill et al 2017). Only 5 of the differentially abundant contigs

261 were significantly increased in CS relative to SVD, none of which showed high enough levels of 262 homology to reliably infer their taxonomy or host (Table S4 \& Table S5). 
duration of the impact has yet to be established. Here we observe a strong correlation between

267

268

269

270

271

272

273

274

275

276

277

278

279

280

281

282

283 birth mode and diversity of the gut virome at one year of age. This may indicate that vertical transmission of viral communities may help shape the early life microbiome. In theory, the ability of the virome to predate on bacterial hosts could increase bacterial diversity, and thus assist overall community fitness. However, before causation can be established this phenomena will need to be characterized both in animal models and in larger human cohorts incorporating longitudinal sample collection. Future studies of gut virome composition and diversity in the first years of life should also consider birth mode as a potential confounding factor.

\section{Data availability}

The raw sequence data has been deposited in the NCBI Sequence Read Archive under the accession number SRP106048. Accession numbers for each individual subject are available in Table S1. FastQC reports, R code, assembled sequences, BLASTn output against the nt database, taxonomic assignments $16 \mathrm{~S}$ sequences and count tables for both virome and 16S rRNA data were deposited in FigShare and are available at https://figshare.com/s/3020d1009f59db059732.

\section{References}

Bolger AM, Lohse M, Usadel B (2014). Trimmomatic: a flexible trimmer for Illumina sequence data. Bioinformatics 30: 2114-2120.

Breitbart M, Hewson I, Felts B, Mahaffy JM, Nulton J, Salamon P, Rohwer F (2003). Metagenomic analyses of an uncultured viral community from human feces. Journal of bacteriology 185: 6220-6223. 
290

291

292

293

294

295

296

297

298

299

300

301

302

303

304

305

306

307

308

309

310

311

312

313

314

315

316

317

318

319

320

321

322

323

Breitbart M, Haynes M, Kelley S, Angly F, Edwards RA, Felts B, Mahaffy JM, Mueller J, Nulton J, Rayhawk S, Rodriguez-Brito B, Salamon P, Rohwer F (2008). Viral diversity and dynamics in an infant gut. Research in Microbiology 159: 367-373.

Coordinators NR (2016). Database resources of the national center for biotechnology information. Nucleic acids research 44: D7.

Dutilh BE, Cassman N, McNair K, Sanchez SE, Silva GGZ, Boling L, Barr JJ, Speth DR, Seguritan V, Aziz RK, Felts B, Dinsdale EA, Mokili JL, Edwards RA (2014). A highly abundant bacteriophage discovered in the unknown sequences of human faecal metagenomes. Nature Communications 5: 4498.

Grazziotin AL, Koonin EV, Kristensen DM (2016). Prokaryotic virus orthologous groups (pVOGs): a resource for comparative genomics and protein family annotation. Nucleic acids research: gkw975.

Heiman ML, Greenway FL (2016). A healthy gastrointestinal microbiome is dependent on dietary diversity. Molecular metabolism 5: 317-320.

Hill CJ, Lynch DB, Murphy K, Ulaszewska M, Jeffery IB, O’Shea CA, Watkins C, Dempsey E, Mattivi F, Tuohy K, Ross RP, Ryan CA, O' Toole PW, Stanton C (2017). Evolution of gut microbiota composition from birth to 24 weeks in the INFANTMET Cohort. Microbiome 5: 4.

Hill JE, Penny SL, Crowell KG, Goh SH, Hemmingsen SM (2004). cpnDB: a chaperonin sequence database. Genome research 14: 1669-1675.

Hsiao K-L, Wang L-Y, Lin C-L, Liu H-F (2016). New Phylogenetic Groups of Torque Teno Virus Identified in Eastern Taiwan Indigenes. PloS one 11: e0149901.

Hyatt D, Chen G-L, LoCascio PF, Land ML, Larimer FW, Hauser LJ (2010). Prodigal: prokaryotic gene recognition and translation initiation site identification. BMC bioinformatics 11: 119.

Imhann F, Vich Vila A, Bonder MJ, Fu J, Gevers D, Visschedijk MC, Spekhorst LM, Alberts R, Franke L, van Dullemen HM, Ter Steege RWF, Huttenhower C, Dijkstra G1, Xavier RJ, Festen EAM, Wijmenga C, Zhernakova A, Weersma RK (2016). Interplay of host genetics and gut microbiota underlying the onset and clinical presentation of inflammatory bowel disease. Gut. 
Karlsson FH, Tremaroli V, Nookaew I, Bergstrom G, Behre CJ, Fagerberg B, Nielsen J, Bäckhed F (2013). Gut metagenome in European women with normal, impaired and diabetic glucose control. Nature 498: 99-103.

Kopylova E, Noé L, Touzet H (2012). SortMeRNA: fast and accurate filtering of ribosomal RNAs in metatranscriptomic data. Bioinformatics 28: 3211-3217.

Ley RE, Bäckhed F, Turnbaugh P, Lozupone CA, Knight RD, Gordon JI (2005). Obesity alters gut microbial ecology. Proceedings of the National Academy of Sciences of the United States of America 102: 11070-11075.

Lim ES, Zhou Y, Zhao G, Bauer IK, Droit L, Ndao IM, Warner BB, Tarr PI, Wang D, Holtz LR (2015). Early life dynamics of the human gut virome and bacterial microbiome in infants. Nature Medicine 21: 1228.

Love MI, Huber W, Anders S (2014). Moderated estimation of fold change and dispersion for RNA-seq data with DESeq2. Genome biology 15: 550.

Martin M (2011). Cutadapt removes adapter sequences from high-throughput sequencing reads. EMBnet journal 17: pp. 10-12.

McMurdie PJ, Holmes S (2013). phyloseq: an R package for reproducible interactive analysis and graphics of microbiome census data. PloS one 8: e61217.

Menzel P, Ng KL, Krogh A (2016). Fast and sensitive taxonomic classification for metagenomics with Kaiju. Nature communications 7.

Milani C, Duranti S, Bottacini F, Casey E, Turroni F, Mahony J, Belzer C, Delgado Palacio S, Arboleya Montes S, Mancabelli L, Lugli GA, Rodriguez JM, Bode L, de Vos W, Gueimonde M, Margolles A, van Sinderen D, Ventura M (2017). The First Microbial Colonizers of the Human Gut: Composition, Activities, and Health Implications of the Infant Gut Microbiota. Microbiology and Molecular Biology Reviews $\mathbf{8 1 .}$

Minot S, Bryson A, Chehoud C, Wu GD, Lewis JD, Bushman FD (2013). Rapid evolution of the human gut virome. Proc Natl Acad Sci US A 110: 12450-12455. 
361

362

363

364

365

366

367

368

369

370

371

372

373

374

375

376

377

378

379

380

381

382

383

384

385

386

387

388

389

390

391

392

393

NCBI Resource Coordinators (2018). Database resources of the national center for biotechnology information. Nucleic acids research 46: D8-D13.

Norman JM, Handley SA, Baldridge MT, Droit L, Liu CY, Keller BC, Kambal A, Monaco CL, Zhao G, Fleshner P, Stappenbeck TS, McGovern DP, Keshavarzian A, Mutlu EA, Sauk J, Gevers D, Xavier RJ, Wang D, Parkes M, Virgin HW (2015). Disease-specific Alterations in the Enteric Virome in Inflammatory Bowel Disease. Cell 160: 447-460.

Nurk S, Meleshko D, Korobeynikov A, Pevzner PA (2017). metaSPAdes: a new versatile metagenomic assembler. Genome research 27: 824-834.

Oksanen J, Blanchet FG, Kindt R, Legendre P, O'Hara RG, Simpson GL, Solymos P, Stevens $\mathrm{MHH}$, Wagner $\mathrm{H}$ (2007). The vegan package. Community ecology package Available at https://CRAN.R-project.org/package= vegan (accessed 09 April 2018)

Reyes A, Blanton LV, Cao S, Zhao G, Manary M, Trehan I, Smith MI, Wang D, Virgin HW, Rohwer F, Gordon JI (2015). Gut DNA viromes of Malawian twins discordant for severe acute malnutrition. Proc Natl Acad Sci U S A 112: 11941-11946.

Rodriguez JM, Murphy K, Stanton C, Ross RP, Kober OI, Juge N, Avershina E, Rudi K, Narbad A, Jenmalm MC, Marchesi JR, Collado MC (2015). The composition of the gut microbiota throughout life, with an emphasis on early life. Microbial ecology in health and disease 26: 26050.

Roux S, Enault F, Hurwitz BL, Sullivan MB (2015). VirSorter: mining viral signal from microbial genomic data. PeerJ 3: e985.

Roux S, Solonenko NE, Dang VT, Poulos BT, Schwenck SM, Goldsmith DB, Coleman ML, Breitbart M, Sullivan MB (2016). Towards quantitative viromics for both double-stranded and single-stranded DNA viruses. PeerJ 4: e2777.

Spandole S, Cimponeriu D, Berca LM, Mihaescu G (2015). Human anelloviruses: an update of molecular, epidemiological and clinical aspects. Archives of virology 160: 893-908. 
394 Ssemadaali MA, Effertz K, Singh P, Kolyvushko O, Ramamoorthy S (2016). Identification of 395 heterologous Torque Teno Viruses in humans and swine. Scientific reports 6: 26655.

396

397 Sullivan MJ, Petty NK, Beatson SA (2011). Easyfig: a genome comparison visualizer. 398 Bioinformatics 27: 1009-1010.

399

400

401

402

403

404

405

406

407

408

409

410

411

412

413

414

415

416

417

418

419

420

421
R core team (2016). R: A language and environment for statistical computing. Vienna, Austria: $R$ foundation for statistical computing. Available at http:/www.R-project.org/ (accessed 09 April 2018)

Tyschik EA, Shcherbakova SM, Ibragimov RR, Rebrikov DV (2017). Transplacental transmission of torque teno virus. Virology Journal 14.

Weiss S, Xu ZZ, Peddada S, Amir A, Bittinger K, Gonzalez A, Lozupone C, Zaneveld JR, Vázquez-Baeza Y, Birmingham A, Hyde ER, Knight R (2017). Normalization and microbial differential abundance strategies depend upon data characteristics. Microbiome 5: 27.

Wickham H (2016). ggplot2: elegant graphics for data analysis. Springer.

Yarza P, Yilmaz P, Pruesse E, Glockner FO, Ludwig W, Schleifer KH, Whitman WB5, Euzéby J6, Amann R2, Rosselló-Móra R (2014). Uniting the classification of cultured and uncultured bacteria and archaea using 16S rRNA gene sequences. Nature reviews Microbiology 12: 635-645.

Yutin N, Makarova KS, Gussow AB, Krupovic M, Segall A, Edwards RA, Koonin EVl (2017). Discovery of an expansive bacteriophage family that includes the most abundant viruses from the human gut. Nature Microbiology. 


\section{Figure 1 (on next page)}

Classification and abundance of known viral groups in the INFANTMET cohort.

(A): Log relative abundance of classifiable viral groups by the Kaiju amino acid classifier against the NR protein database. (B) Boxplot of the number of detectable homologues of Torque Teno Virus (TTV) ORF1 in each sample by birth mode. (C) Visualized alignment of multiple CrAssphage genomes of infant origin. 


\section{Figure 2 (on next page)}

Alpha and beta diversity measures for virome and 16S rRNA sequence data in the INFANTMET cohort.

PCoAs of unweighted Bray-Curtis distances for the (A) virome and (B) 16S rRNA sequence datasets, respectively. Boxplots of Shannon diversity in the (C) virome and (D) 16S rRNA sequence datasets, respectively. 


\section{DNPANW/irome}
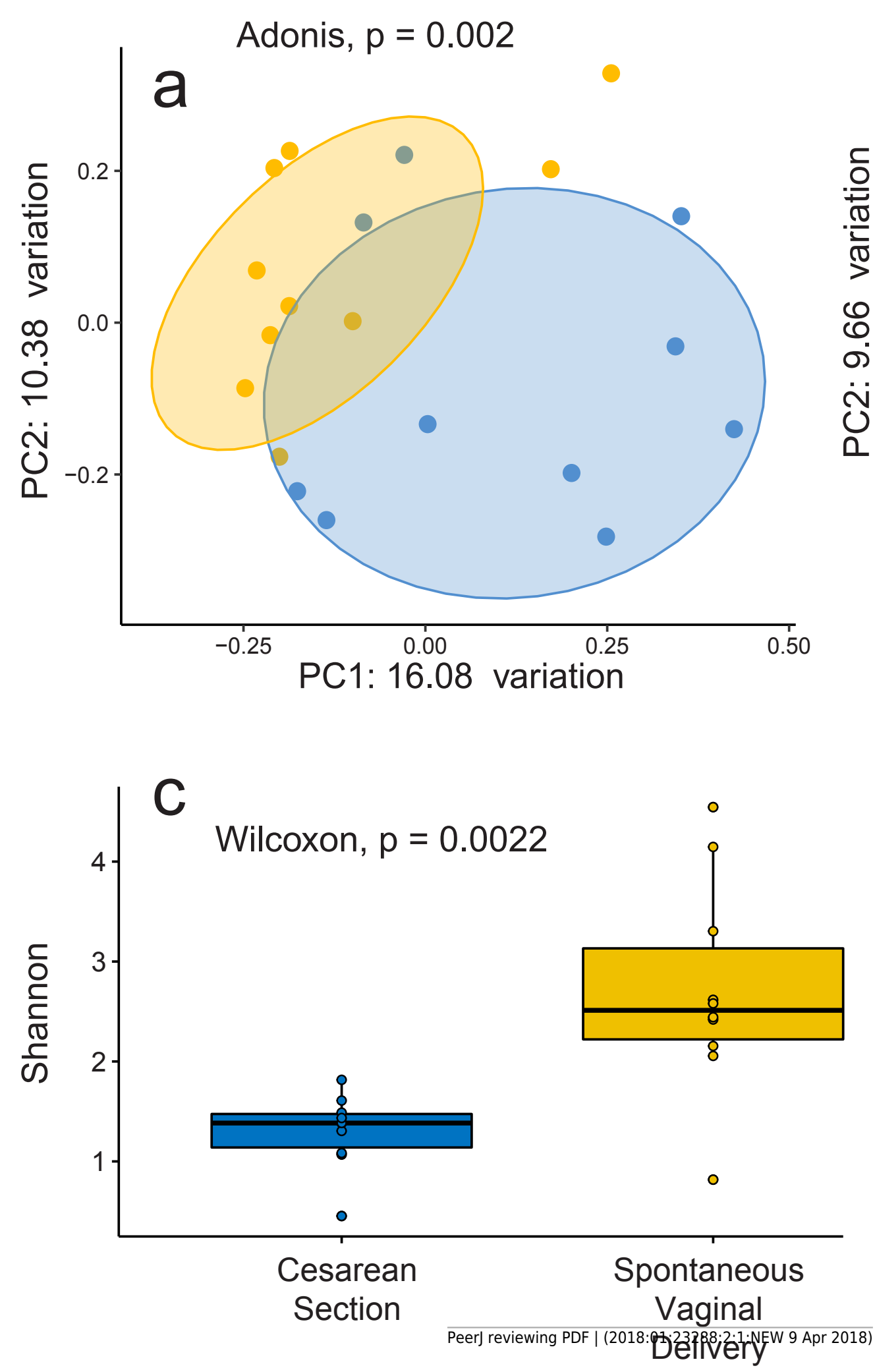
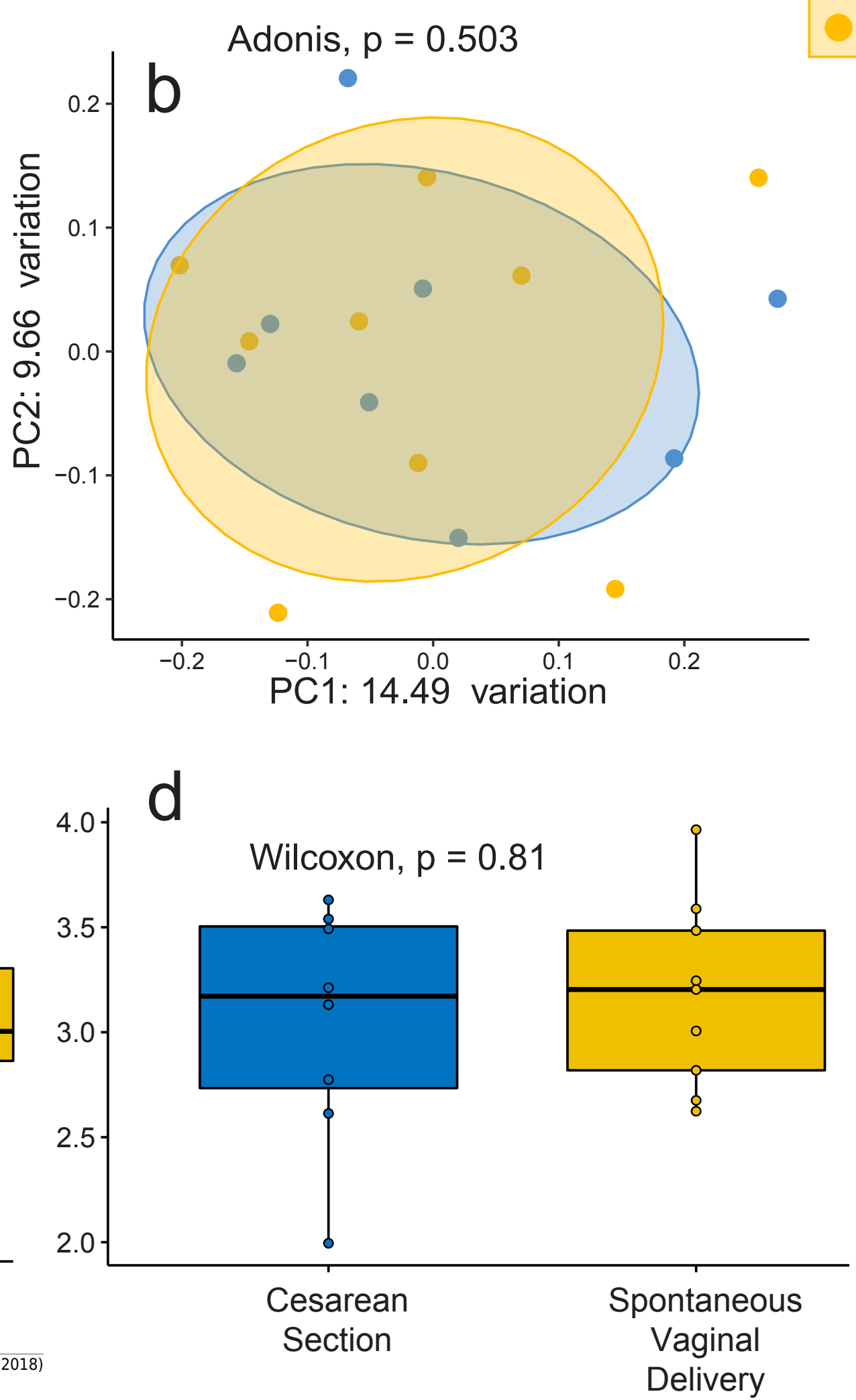